\title{
SC06992, a Protein with $\beta$-Glucuronidase Activity, Complements a Mutation at the absR Locus and Promotes Antibiotic Biosynthesis in Streptomyces coelicolor
}

\author{
Xue-Mei Jin ${ }^{1,2}$, Mu-Yong Choi ${ }^{3}$, Maral Tsevelkhoroloo ${ }^{1}$, Uhnmee Park ${ }^{3}$, Joo-Won Suh ${ }^{1}$, and \\ Soon-Kwang Hong ${ }^{1 *}$ \\ ${ }^{1}$ Department of Bioscience and Bioinformatics, Myongji University, Yongin 17058, Republic of Korea \\ ${ }^{2}$ Characteristic Industry Development Center of Yanbian, Jilin Province, P.R. China \\ ${ }^{3}$ Department of Biotechnology. The University of Suwon, Suwon 18323, Republic of Korea
}

\begin{abstract}
Streptomyces coelicolor is a filamentous soil bacterium producing several kinds of antibiotics. S. coelicolor abs8752 is an abs (antibiotic synthesis deficient)-type mutation at the absR locus; it is characterized by an incapacity to produce any of the four antibiotics synthesized by its parental strain J1501. A chromosomal DNA fragment from S. coelicolor J1501, capable of complementing the abs phenotype of the abs8752 mutant, was cloned and analyzed. DNA sequencing revealed that two complete ORFs (SCO6992 and SC06993) were present in opposite directions in the clone.

Introduction of SCO6992 in the mutant strain resulted in a remarkable increase in the production of two pigmented antibiotics, actinorhodin and undecylprodigiosin, in S. coelicolor J1501 and abs8752. However, introduction of SCO6993 did not show any significant difference compared to the control, suggesting that SCO6992 is primarily involved in stimulating the biosynthesis of antibiotics in S. coelicolor. In silico analysis of SCO6992 (359 aa, $39.5 \mathrm{kDa}$ ) revealed that sequences homologous to SCO6992 were all annotated as hypothetical proteins. Although a metalloprotease domain with a conserved metal-binding motif was found in SCO6992, the recombinant rSCO6992 did not show any protease activity. Instead, it showed very strong $\beta$-glucuronidase activity in an API ZYM assay and toward two artificial substrates, $p$-nitrophenyl- $\beta$-D-glucuronide and AS-BI- $\beta$-D-glucuronide. The binding between rSCO6992 and $\mathrm{Zn}^{2+}$ was confirmed by circular dichroism spectroscopy. We report for the first time that SCO6992 is a novel protein with $\beta$-glucuronidase activity, that has a distinct primary structure and physiological role from those of previously reported $\beta$-glucuronidases.
\end{abstract}

Keywords: Streptomyces coelicolor, SCO6992, actinorhodin, undecylprodigiosin, $\beta$-glucuronidase

Received: August 2, 2021 Accepted: September 23, 2021 First published online: September 25, 2021

*Corresponding author Phone: +81-31-330-6198 E-mail: skhong@mju.ac.kr

pISSN 1017-7825 eISSN 1738-8872

Copyright $(2021$ by the authors. Licensee KMB. This article is an open access article distributed under the terms and conditions of the Creative Commons Attribution (CC BY) license.

\section{Introduction}

Streptomycetes is a group of gram-positive and filamentous soil bacteria with a unique and complex differentiation process that includes simultaneous morphological (sporulation) and physiological (secondary metabolism) differentiation. Many scientific findings have elucidated that the two differentiation processes are genetically closely related and thus controlled concurrently by many regulatory factors. Generally, genes for secondary metabolite formation and morphological differentiation are clustered in the chromosome, and their expression is precisely controlled by a pathway-specific regulatory gene(s) within the cluster as well as by global regulatory genes [1-3].

Several classes of mutants that simultaneously block the synthesis of more than one antibiotic constitute a good genetic evidence of a global regulatory system. Streptomyces coelicolor has been known to produce four genetically and structurally distinct antibiotics: the pigmented antibiotics actinorhodin and undecylprodigiosin and the unpigmented antibiotics methylenomycin and $\mathrm{CDA}\left(\mathrm{Ca}^{2+}\right.$-dependent antibiotic) [4]. According to an S. coelicolor genome sequence analysis, 18 gene clusters are expected to code exclusively for enzymes characteristic of its secondary metabolism [5]. abs (antibiotic synthesis deficient) mutants, for example, affect global regulatory genes involved only in physiological differentiation. Among them, $a b s$ mutations $a b s A^{-}, a b s B$, and $a b s C$ were found to lack all four antibiotics, but retained normal sporulation. The molecular nature of the absA1/A2 gene products that can restore the bacterial ability for biosynthesizing all four antibiotics was identified as a two-component regulatory system, composed by the sensor histidine kinase AbsA1 and the aspartate kinase response regulator AbsA2 [4]. The phosphorylated AbsA2 is known to act as a global negative regulator of antibiotic biosynthesis in $S$. 
coelicolor [6]. Besides, the global defect in antibiotic synthesis in the abs $B^{-}$mutant was due to a deficiency in RNase III $(r n c)$ [7] which regulates the production of antibiotics through its double strand-specific endoRNase activity [8]. Finally, the absClocus exerts pleiotropic, zinc-dependent effects on antibiotic biosynthesis [9]. Interestingly, it was shown that the production of actinorhodin and undecylprodigiosin was abolished when $a b s C$ was deleted, but this phenomenon was only observed when zinc concentrations were low. AbsC also represses the expression of the coelibactin biosynthetic gene cluster by binding to an operator sequence adjacent to the zinc-responsive regulatory protein Zur binding site [9]. Although these three mutations such as $a b s A$, abs $B$, and $a b s C$ are categorized into the same abs mutation, the molecular properties of them are quite different, implying that the control machinery for secondary metabolism in Streptomyces is extraordinarily complex [10].

We previously reported on the genetic analysis of a new abs-like mutation at the $a b s R$ locus of the mutant strain S. coelicolor abs8752 [11]. The absR mutation was complemented with multiple copies of the actII-ORF4 gene encoding for a pathway specific regulator for actinorhodin biosynthesis [3] and by the afs $R$ gene encoding a global regulator for morphological and physiological differentiation [12], but not with $a b s A$. Therefore, as absR was considered an abs-type mutation distinct from $a b s A^{-}$or $a b s B^{-}$mutations [11], the chromosomal DNA fragment that could complement strain abs8752 for antibiotic biosynthesis was cloned from its parental strain, S. coelicolor J1501. In this fragment, two complete ORFs, namely ORF-1 (equivalent to SCO6992) and ORF-2 (equivalent to SCO6993), were identified. In this report, we describe the functional analysis of the SCO6992 gene; a gene that is able to restore and stimulate antibiotic biosynthesis in S. coelicolor.

\section{Materials and Methods}

\section{Bacterial Strains and Plasmids}

S. coelicolor $\mathrm{J} 1501$ (his $1^{-}$uraA1 $1^{-}$strA1 $\mathrm{SCP}^{-} \mathrm{SCP}^{-} \mathrm{Pgl}^{-}$) derived from S. coelicolor A3(2) and Streptomyces lividans TK24 were obtained from the John Inns Institute, U.K. [13]. S. coelicolor abs8752 (an absR mutant that was selected as a mutant blocked in antibiotic biosynthesis after treating J1501 with $N$-methyl- $N^{\prime}$-nitro- $N$ nitrosoguanidine) was kindly given by Dr. Wendy Champness from Michigan State University [14]. The Escherichia coli-Streptomyces shuttle vector pWHM3 [15] was used for cloning and expression in Streptomyces spp.. E. coli JM109 was used for genetic manipulations.

\section{Media and Culture Conditions}

E. coli was maintained on M9 minimal agar and routinely cultured in Luria broth [13] at $37^{\circ} \mathrm{C}$ with agitation. Streptomyces strains were maintained on $\mathrm{R} 2 \mathrm{YE}$ agar or liquid medium at $30^{\circ} \mathrm{C}$ [13]. For the cultivation of S. coelicolor strains, histidine and uracil were used as supplements at final concentrations of 50 and $7.5 \mu \mathrm{g} / \mathrm{ml}$, respectively [11].

\section{Enzymes and Chemicals}

Restriction endonucleases and other DNA modifying enzymes were purchased from DyneBio Inc. (Korea). Unless specified, any other fine chemical was from Sigma-Aldrich Corporation (USA).

\section{DNA Manipulation and Transformation}

DNA preparation and manipulation were performed by the methods described by Kieser et al. [13]. All kits and enzymes were used according to the manufacturer's recommendations. Preparation of competent $E$. coli cells and transformations were performed as described [13]. Protoplast preparation and PEG-mediated transformation of Streptomyces cells were carried out as described [13]. Streptomyces transformants were selected by overlaying with $0.6 \%$ soft agar containing $25 \mu \mathrm{g} / \mathrm{ml}$ of thiostrepton.

\section{Cloning of a DNA Fragment with absR-Complementing Activity}

S. coelicolor J1501 was cultured in R2YE medium and used for chromosomal DNA isolation, as described previously [16]. To clone the $a b s R^{-}$complementing DNA fragment, the chromosomal DNA was partially digested

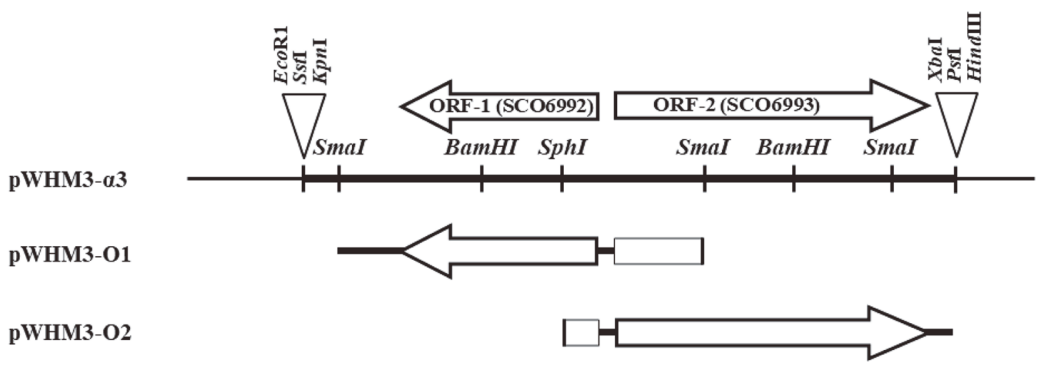

Fig. 1. Schematic representation of the genes within the insert in pWHM3- $\alpha 3$ and fragments used in the construction of expression vectors for each open reading frame (ORF). Arrows indicate individual ORFs, and arrowheads mark the corresponding stop codons. DNA fragments derived from S. coelicolor $\mathrm{J} 1501$ are indicated by thick lines, and vector fragments, including the multiple cloning site, are depicted by thin lines. For the construction of pWHM3-O1 and pWHM3-O2, DNA fragments digested with SmaI-SmaI or SphI-PstI, respectively, were subcloned into pWHM3, an E. coliStreptomyces shuttle vector. 
with restriction enzyme Sau3A1, and separated elctrophoretically on a 1\% agarose gel. The pool of 4-6 kb DNA fragments recovered from the gel was ligated with the pWHM3 vector digested with BamHI to construct a mini library by shotgun cloning. Ligated plasmids were transformed into S. coelicolor abs8752. Among the 1,650 transformants, one colony with an $a b s^{+}$phenotype (pigment production) was selected to isolate the recombinant plasmid, pWHM3- $\alpha 3$, which carried a 3,919-bp insert (GenBank accession number; AF136167) that included two ORFs, according to DNA sequencing analysis (Fig. 1). The two ORFs, ORF-1 and ORF-2, were identified as SCO6992 and SCO6993 annotated from the S. coelicolor A3(2) genome sequence [5], respectively.

\section{Quantitative Analysis of Antibiotic Production}

Each ORF was individually subcloned. A SmaI fragment, 1,894 bp long, containing the entire SCO6992 including its promoter region was subcloned into pWHM3 to yield pWHM3-O1. In addition, a SphI-PstI digested fragment, 2,711 bp long, containing the entire SCO6993 and its promoter region was cloned into pWHM3 to yield pWHM3-O2 (Fig. 1). The capacity of producing pigmented antibiotics actinorhodin (blue) and undecylprodigiosin (red) by S. coelicolor and S. lividans TK24 transformants could be easily checked with the naked eye on a solid medium. Quantitation of actinorhodin and undecylprodigiosin in liquid culture was performed as previously described [12]. Briefly, an exponential phase culture of S. coelicolor was transferred to $100 \mathrm{ml}$ of R2YE medium containing $25 \mu \mathrm{g} / \mathrm{ml}$ of thiostrepton in a $500 \mathrm{ml}$ baffled flask, and incubated at $30^{\circ} \mathrm{C}$ on a reciprocal shaker. At indicated intervals, $5 \mathrm{ml}$ aliquots of the culture broth were extracted with $5 \mathrm{ml}$ of chloroform for $30 \mathrm{~min}$ at $20^{\circ} \mathrm{C}$, with shaking. Then, $5 \mathrm{ml}$ of $1 \mathrm{~N} \mathrm{NaOH}$ was added, and the tubes were vortexed and spun in a microcentrifuge for $15 \mathrm{~s}$. Actinorhodin, distributed to the aqueous phase, had a blue color at alkaline $\mathrm{pH}$, and was quantified by measuring the absorbance at $640 \mathrm{~nm}\left(\mathrm{~A}_{640}\right)$. Undecylprodigiosin had a yellow color at alkaline $\mathrm{pH}$ and remained in the chloroform layer; it was acidified with $\mathrm{HCl}$, and turned to red at acidic $\mathrm{pH}$. It was quantified by measuring the $\mathrm{A}_{530}$. The concentrations of actinorhodin and undecylprodigiosin were calculated using the molar extinction coefficients $\varepsilon_{640}=25,320 \mathrm{M}^{-1} \mathrm{~cm}^{-1}$ and $\varepsilon_{530}=100,150 \mathrm{M}^{-1} \mathrm{~cm}^{-1}$, respectively.

\section{Expression and Purification of the MBP-SCO6992 Fusion Protein in E. coli}

The pMAL-c2x vector was used to express the SCO6992 protein in E. coli. The SCO6992 DNA fragment (1,080 bp long) was amplified by PCR as previously described [17] using primers SCO6992-forward (5'GTCGACATGACCGCACGCTACTGCTCG-3', the SalI site is underlined) and SCO6992-reverse (5'AAGCTTTCACCAGTACATGACCGCCGTC-3', the HindIII site is underlined). The amplified fragment was then cloned into pMAL-c2x digested with SalI/HindIII restriction enzymes. The resulting plasmid, pMAL-c2xSCO6992, was designed to express SCO6992 fused to MalE maltose binding protein (MBP). E. coli JM109/pMALc2x-SCO6992 cells were inoculated in $1 \mathrm{~L}$ of rich medium (Luria broth containing $0.2 \%$ glucose) with ampicillin $(50 \mu \mathrm{g} / \mathrm{ml})$ and cultured at $37^{\circ} \mathrm{C}$ with shaking. When the optical density $\left(\mathrm{OD}_{600}\right)$ of the culture broth was 0.4 , isopropyl-D-thiogalactopyranoside $(0.3 \mathrm{mM})$ was added to induce SCO6992 expression. After culturing for $3 \mathrm{~h}$, the cells were harvested by centrifugation $\left(7,000 \times g, 10 \mathrm{~min}, 4^{\circ} \mathrm{C}\right)$ and resuspended in buffer $\mathrm{A}(20 \mathrm{mM}$ Tris- $\mathrm{HCl}$ $\mathrm{pH} 7.4,200 \mathrm{mM} \mathrm{NaCl}, 1 \mathrm{mM}$ EDTA, $10 \mathrm{mM} \beta$-mercaptoethanol). Cells were then disrupted by sonication and centrifuged at $15,000 \times g$ for $30 \mathrm{~min}$ at $4^{\circ} \mathrm{C}$ to prepare a cell-free lysate that was used for the purification of the MBPSCO6992 fusion protein (MBP-SCO6992) by using an MBP.Bind Agarose Resin (ELPIS Biotech, Korea). The purified MBP-SCO6992 protein, eluted with buffer A containing $10 \mathrm{mM}$ maltose, was concentrated using an Amicon ultra centrifugal filter $50 \mathrm{~K}$ device, followed by a buffer exchange for $10 \mathrm{mM}$ Tris- $\mathrm{HCl}$ ( $\mathrm{pH}$ 7.9) for further experimentation. The purified protein was analyzed by $0.1 \%$ sodium dodecyl sulfate- $10 \%$ polyacrylamide gel electrophoresis (SDS-PAGE).

\section{Circular Dichroism (CD) Spectroscopy Analysis}

To investigate the complexes formed between the MBP-SCO6992 protein and different metal ions, CD spectroscopy analysis was performed using a J-815 spectropolarimeter (Jasco Corp., Japan), using a wavelength range of $200-260 \mathrm{~nm}$, and a quartz cell with a path length of $0.1 \mathrm{~mm}$ [18]. Scanning was performed 10 times per sample at a scan rate of $100 \mathrm{~nm}$ per min. Measurements were done at $20^{\circ} \mathrm{C}$, with $1 \mu \mathrm{g} / \mu \mathrm{l}$ protein and $2 \mathrm{mM}$ metal ion (either $\mathrm{Fe}^{2+}, \mathrm{Zn}^{2+}, \mathrm{Mn}^{2+}, \mathrm{Ca}^{2+}, \mathrm{Mg}^{2+}$, or $\mathrm{Cu}^{2+}$ ).

\section{Protease Activity Determination}

A skim milk hydrolysis assay was performed to detect protease activity. Briefly, $20 \mu$ l of purified MBP-SCO6992 was dropped onto a $1 \%$ skim milk agar plate, and the formation of a clear zone was observed after incubation at $37^{\circ} \mathrm{C}$ for $24 \mathrm{~h}$.

The azocasein hydrolysis method was then used to measure the total protease activity of the purified MBPSCO6992 as follows: $120 \mu \mathrm{l}$ of protein was mixed with $480 \mu \mathrm{l}$ of $1 \%$ azocasein solution in $25 \mathrm{mM}$ Tris-HCl buffer ( $\mathrm{pH} 8.0)$, and left to react at $37^{\circ} \mathrm{C}$ for $30 \mathrm{~min}$. Then, the reaction was terminated by adding $600 \mu \mathrm{l}$ of a $10 \%(\mathrm{w} / \mathrm{v})$ trichloroacetic acid solution. After centrifugation at $15,000 \times \mathrm{g}$ for $10 \mathrm{~min}, 800 \mu \mathrm{l}$ of the supernatant was taken and mixed with $200 \mu \mathrm{l}$ of $1.8 \mathrm{~N} \mathrm{NaOH}$. The concentration of azo dye, a hydrolysis product, was measured spectrophotometrically at $420 \mathrm{~nm}\left(\mathrm{~A}_{420}\right)$. The effect of metal ions on protease activity were also investigated by adding various metal ions to the reaction mixture.

\section{Enzyme Activity Analysis}

An API ZYM kit (bioMérieux, France) was used to investigate the enzymatic activity of the purified protein. Following the manufacturer's instructions, $200 \mu$ l of purified protein was added to each plate well together with the 
corresponding reaction reagents. The presence or absence of enzymatic activity was determined according to the resultant color change.

\section{Measurement of $\beta$-Glucuronidase Activity}

To measure the $\beta$-glucuronidase activity of the purified protein, an artificial substrate $p$-nitrophenyl- $\beta$-Dglucuronide was used [19]. The reaction mixture $(600 \mu \mathrm{l})$ contained $83 \mathrm{mM}$ acetate buffer $(\mathrm{pH} 4.5), 1.67 \mathrm{mM} p$ nitrophenyl- $\beta$-D-glucuronide, and $200 \mu$ of purified protein (after the final concentration step). After incubating the reaction mix at $37^{\circ} \mathrm{C}$ for $1 \mathrm{~h}, 400 \mu \mathrm{l}$ of $2.5 \mathrm{M}$ 2-amino-2-methyl-1,3-propanediol was added to stop the reaction, and the concentration of $p$-nitrophenol, a hydrolysis product, was measured at $415 \mathrm{~nm}\left(\mathrm{~A}_{415}\right)$.

For $\beta$-glucuronidase zymographic analysis, protein samples were loaded onto a $9 \%$ native polyacrylamide gel. Electrophoresis was performed in $25 \mathrm{mM}$ Tris- $\mathrm{HCl}$ ( $\mathrm{pH}$ 8.1) buffer containing $192 \mathrm{mM}$ glycine, at $4^{\circ} \mathrm{C}$ and $160 \mathrm{~V}$ for $2 \mathrm{~h}$. After washing twice with triple distilled water, the gel was immersed in $100 \mathrm{~mL}$ of $0.2 \mathrm{M}$ sodium acetate buffer (pH 5.2) containing $40 \mathrm{mg}$ of naphthol AS-BI- $\beta$-D-glucuronide (an artificial substrate) and $40 \mathrm{mg}$ of Fast Garnet, and the color change on the zymogram was observed [20].

\section{Results}

\section{Complementation of the $a b s R^{-}$Mutation by the pWHM3-a3 Clone}

S. coelicolor abs8752 was used as the host strain to identify the gene complementing the abs phenotype. First, one thiostrepton-resistant transformant with $a b s^{+}$phenotype on an agar plate was selected, and the recombinant plasmid (named pWHM3-a3) carrying a 4-kb insert was isolated from it (Fig. 1).

pWHM3- $\alpha 3$ was able to restore actinorhodin and undecylprodigiosin biosynthesis in S. coelicolor abs8752 on R2YE plates (Fig. 2A). Judging by the color of the bacterial growth, undecylprodigiosin (red color) and

A

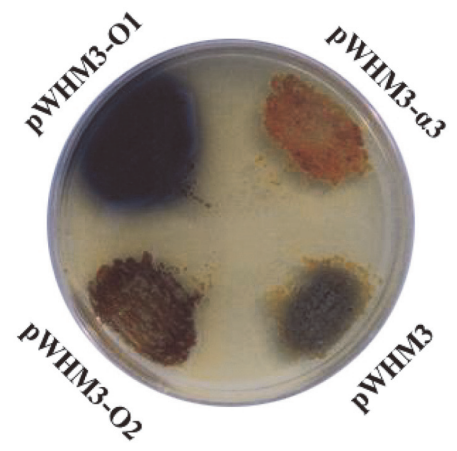

S. coelicolor $\mathbf{J 1 5 0 1}$

B

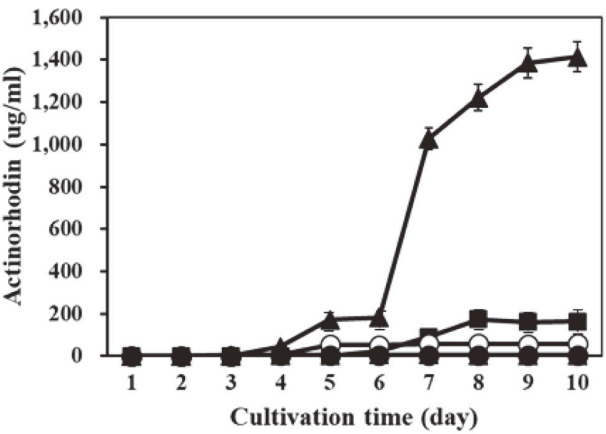

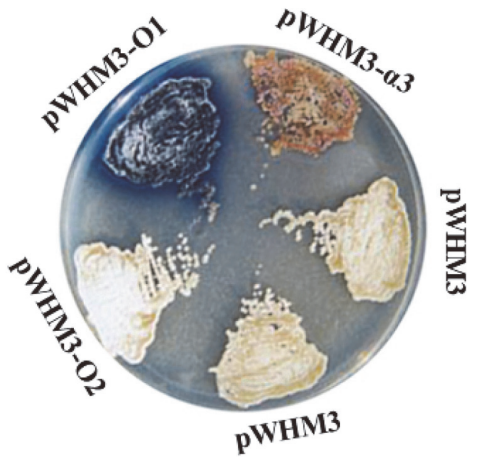

S. coelicolor abs8752

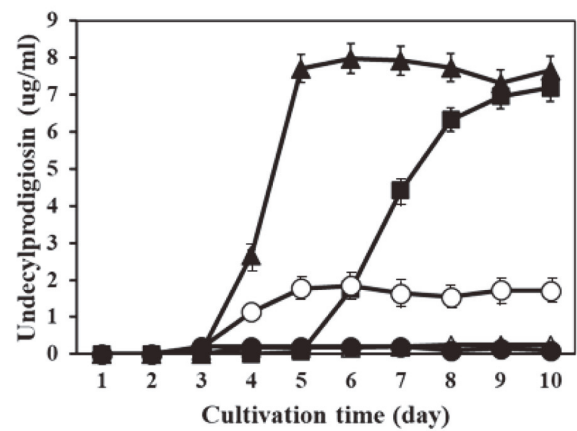

Fig. 2. Effect of cloned DNA fragments on pigmented antibiotic production in Streptomyces coelicolor. (A) Production of pigmented antibiotics by S. coelicolor J1501 (left) and S. coelicolor abs8752 (right) transformants carrying different plasmids. Transformants were grown on R2YE plates for 5 days before being photographed. Compared to wild type $S$. coelicolor J1501/pWHM3, S. coelicolor abs8752/pWHM3 did not produce any pigment. However, S. coelicolor abs8752/ pWHM3- $\alpha 3$ shows a dark-reddish color, indicating a restoration in the production of pigmented antibiotics. Complementation with individual ORFs showed that only ORF-1 (plasmid pWHM3-O1) could remarkably stimulate antibiotic production (especially actinorhodin pigment) in both strains. (B) Pigmented antibiotic production by S. coelicolor cultured in R2YE broth. Culture samples were taken at regular intervals of time and processed, as described in Materials and Methods. Then, quantification of actinorhodin (left) and undecylprodigiosin (right) was done by measuring the absorbance at 640 and $530 \mathrm{~nm}$, respectively. $\bigcirc$, S. coelicolor J1501/pWHM3; - S. coelicolor abs8752/pWHM3; $\boldsymbol{\square}$, S. coelicolor abs8752/ pWHM3-a3; $\Delta$, S. coelicolor abs8752/pWHM3-O1; $\triangle$, S. coelicolor abs8752/pWHM3-O2. All experiments were repeated at least 3 times, and their average values were calculated. 
actinorhodin (blue color) were mixed, though the final color was more similar to that of undecylprodigiosin than to that of actinorhodin. The transformant also showed that its ability to produce the non-pigmented antibiotics methylenomycin and CDA was restored (data not shown). This result strongly supports that the cloned fragment has the ability to complement the pleiotropic antibiotic production deficiency of S. coelicolor abs 8752 .

\section{Molecular Analysis of the pWHM3- $\alpha 3$ Clone}

Nucleotide sequencing revealed that two complete ORFs (ORF-1 and ORF-2), transcribed in opposite directions, were included in the 3,919-bp insert (GenBank accession number: AF136167) of pWHM3-a3. The genes encoding ORF-1 (protein_id: AAF19103.1, 359 amino acids, $39.5 \mathrm{kDa}$ ) and ORF-2 (protein_id: AAF19104.1, 606 amino acids, $65.7 \mathrm{kDa}$ ) were registered in GenBank as absR1 and $a b s \mathrm{R} 2$, respectively, in the sense that they complemented the abs R mutation.

\section{Chromosomal Location of the ORF-1 and ORF-2 in S. coelicolor}

We previously reported that the absR locus exists near absA locus on the chromosomal DNA of $S$. coelicolor. Based on the genomic sequence of S. coelcicolor A3(2), AbsA1 (SCO3225) and AbsA2 (SCO3226) were located between the base sequences 3536945 and 3539347, and ORF-1 (SCO6992) and ORF-2 (SCO6993) were placed between 7759825 and 7762803 of chromosomal DNA. Therefore, it was assumed that absA1/A2 and putative $a b s \mathrm{R} 1 / \mathrm{R} 2$ are separated by more than 4.2 million bp on the chromosome. In addition, the nucleotide sequencing of the DNA fragments revealed that $S$. coelicolor J1501 and abs8752 strains had the same nucleotide sequence in ORF- 1 and ORF- 2 coding region. These results clearly indicate that although ORF-1 and ORF-2 can restore the antibiotic-producing ability of $a b s \mathrm{R}$ mutant, they are different from the actual gene corresponding to the $a b s \mathrm{R}$ locus. Therefore, we marked SCO6992 and SCO6993 assigned by genomic sequencing for ORF-1 and ORF-2, respectively.

\section{Gene Dosage Effect of SCO6992 and SCO6993 on Antibiotics Production}

The introduction of pWHM3-O1 containing SCO6992 in S. coelicolor abs8752 and S. coelicolor J1501 could restore and remarkably stimulate pigment production (mainly blue) on R2YE plates, respectively; pigment production was not observed among transformants carrying either pWHM3-O2 (containing SCO6993) or pWHM3 (Fig. 2A).

When the amount of actinorhodin and undecylprodigiosin produced in R2YE liquid media at day 10 was compared, it was seen that $S$. coelicolor abs8752/pWHM3-O1 produced 24.2 times more actinorhodin than transformants with either pWHM3 or pWHM3-O2, and 8.6 times more actinorhodin than that produced by S. coelicolor abs8752/pWHM3- $\alpha 3$ (Fig. 2B, left). Moreover, the amount of undecylprodigiosin produced by S. coelicolor abs8752/pWHM3-O1 was slightly higher than that produced by S. coelicolor abs8752/pWHM3- $\alpha 3$, and at least 4 times higher than those of the transformants with either pWHM3 or pWHM3-O2 (Fig. 2B, right). This fact clearly indicates that the SCO6992 gene is responsible for inducing the biosynthesis of actinorhodin and undecylprodigiosin in S. coelicolor strains. Interestingly, the stimulatory effect by SCO6992 on antibiotic production (especially on actinorhodin) was severely suppressed by the presence of SCO6992 as shown in S. coelicolor abs8752 and S. coelicolor J1501 transformed with pWHM3-a3, implying that SCO6993 may be a repressor.

\section{In silico Analysis of SCO6992}

The top 100 genes with high homology to SCO6992, resulting from a Blastp search (BLAST: Basic Local Alignment Search Tool (nih.gov)), belonged to Streptomyces species; their identity ranged between $77 \%$ and $99 \%$. However, they are all annotated as hypothetical proteins and their functions have not been elucidated. For example, SCO6992 has 99\% identity with the unidentified AIJ11880.1 protein (361 aa) from S. lividans TK24. Moreover, many hypothetical proteins from various gram-positive and gram-negative bacteria and even fungi were also listed as homologous to SCO6992 with lower scores, suggesting that SCO6992 orthologue is widely distributed in bacteria and other organisms.

Interestingly, SCO6992 has an MMP-like sub-family 3 domain (cd04327), common in zinc-dependent metalloproteases, in the region spanning Ala-33 through Pro-254, with an E-value of $2.36 \times \mathrm{e}^{-88}$ [21]. The MMPlike sub-family 3 domain is constituted by a group of bacterial and fungal metalloprotease domains whose structure is similar to those of matrix metalloproteases and astacin (Fig. 3A). Additionally, SCO6992 has a wellconserved metal binding domain composed of three His-residues that are important elements of the active site of metalloproteases (Fig. 3B) [22].

\section{Expression and Purification of an MBP-SCO6992 Fusion Protein}

To investigate its biochemical function, we tried to express SCO6992 in E. coli in various ways. Due to the difficulty in forming inclusion bodies of the protein alone, an MBP-SCO6992 fusion protein was constructed, by linking SCO6992 (39.5 kDa) to MBP (42.5 kDa), to obtain a soluble form in E. coli. The soluble MBP-SCO6992 fusion protein was successively purified by amylose affinity column chromatography. The purified protein had an approximated molecular weight of $82 \mathrm{kDa}$, as predicted (Fig. 4A).

\section{Analysis of the Metal Binding Ability of the MBP-SCO6992 Protein \\ Whether SCO6992, which has a metal binding motif, could bind metal ions was investigated using CD spectroscopy. The MBP-SCO6992 protein showed its minimum CD value (mdeg) between 210 and $222 \mathrm{~nm}$.}




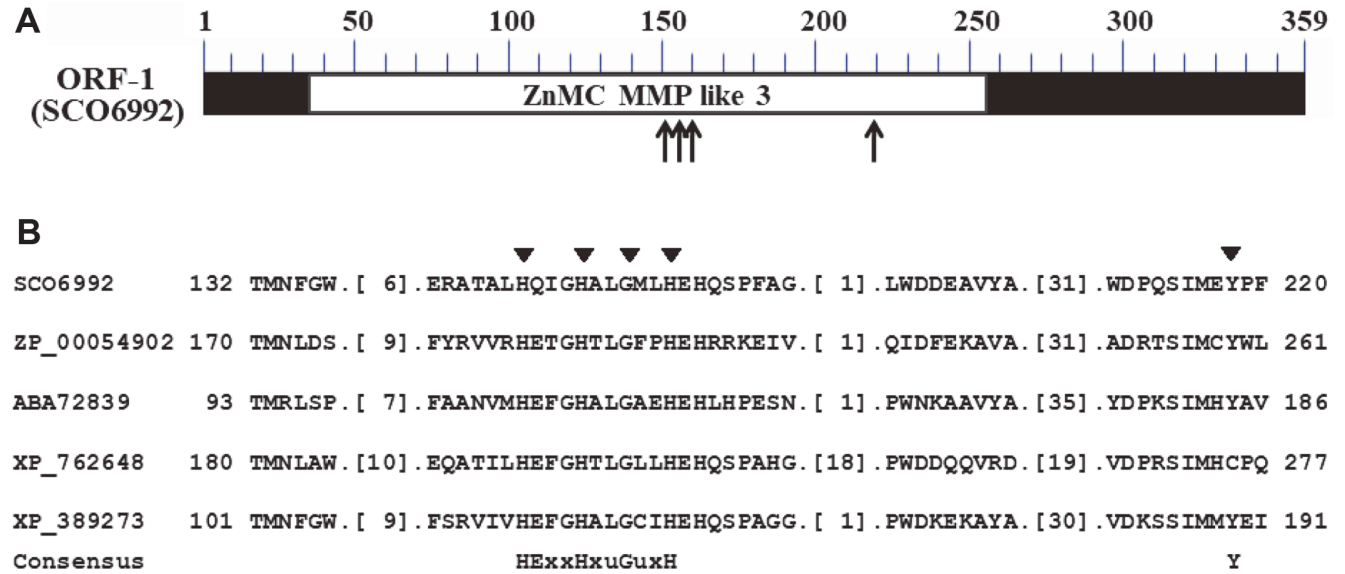

Fig. 3. Conserved metalloprotease domain in SCO6992 and sequence alignment for identifying its metal binding motif. (A) Schematic representation of the zinc-dependent metalloprotease (MMP-like sub-family 3) domain found in SCO6992. The conserved region is represented by an open box, whereas the most highly conserved region, bearing three His and one Tyr residues, is indicated by arrows. (B) Alignment of several amino acid sequences to identify the putative metal binding motif in SCO6992. The metal binding domain cd04327, with the conserved sequence HExxHxuGuxH $(u=$ bulky hydrophobic), has been reported in various zinc-dependent metalloproteases. The most conserved residues, i.e., three His, one Gly, and one Tyr residues, are indicated with black triangles. Aligned proteins belong to Magnetospirillum magnetotacticum MS-1 (ZP_00054902), Pseudomonas fluorescens Pf0-1 (ABA72839), Ustilago maydis 521 (XP_762648), and Fusarium graminearum PH-1 (XP_389273)

A

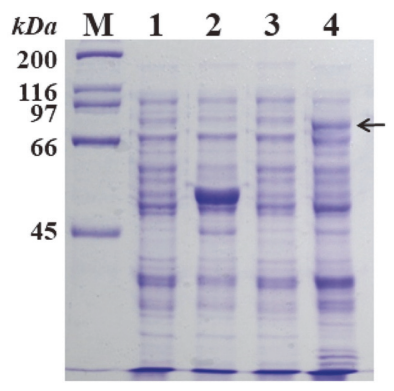

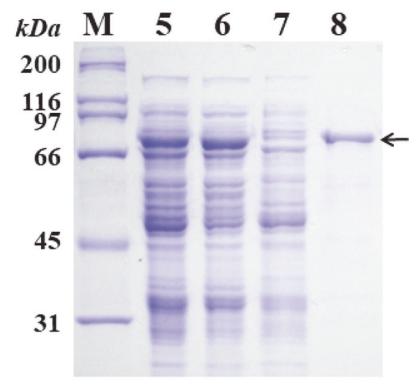

B

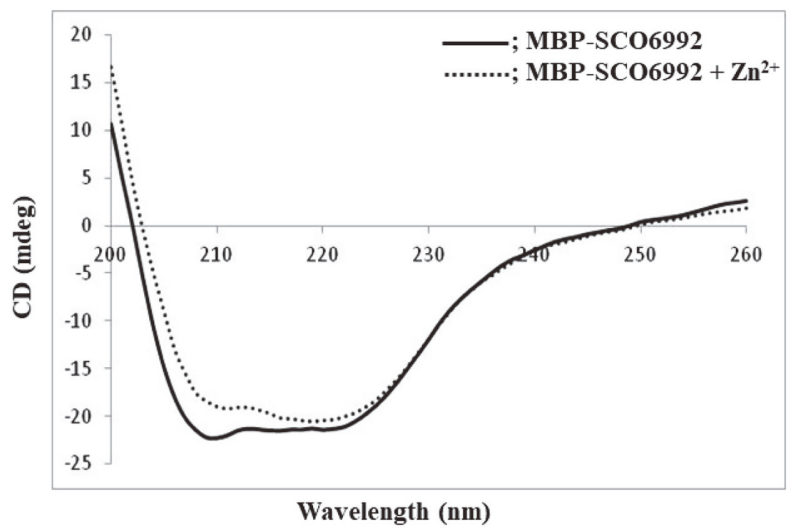

Fig. 4. Purification and confirmation of metal-binding activity of the MBP-SCO6992 recombinant protein. (A) Sodium dodecyl sulfate-polyacrylamide gel electrophoresis of proteins during purification by affinity chromatography. MBP-SCO6992 was overexpressed in E. coli JM109/pMAL-c2x-SCO6992 after IPTG induction (lanes 1-4, left). Then, the recombinant protein was purified from the cell-free extract by an amylose-affinity column chromatography (lanes 5-8, right). The MBP-SCO6992 protein is indicated by arrows. Lanes: M, molecular weight size markers; 1, E. coli JM109/pMAL-c2x before induction; 2, E. coli JM109/pMAL-c2x after induction; 3, E. coli JM109/pMAL-c2x-SCO6992 before induction; 4, E. coli JM109/pMAL-c2x-SCO6992 after induction; 5, cell-free extract of E. coli JM109/pMAL-c2x-SCO6992 after induction; 6, sample after being passed through column; 7, sample after washing the column; 8, purified MBP-SCO6992 protein. (B) Circular dichroism (CD) spectroscopy of MBP-SCO6992 in the presence of $\mathrm{ZnCl}_{2}$. Scanning was performed at a range of 200 and $260 \mathrm{~nm}$ and a scan rate of $100 \mathrm{~nm}$ per minute. 
A

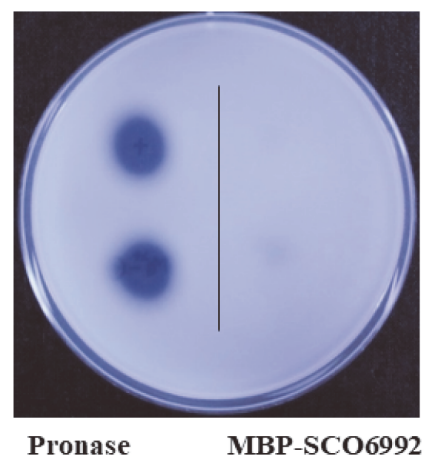

B

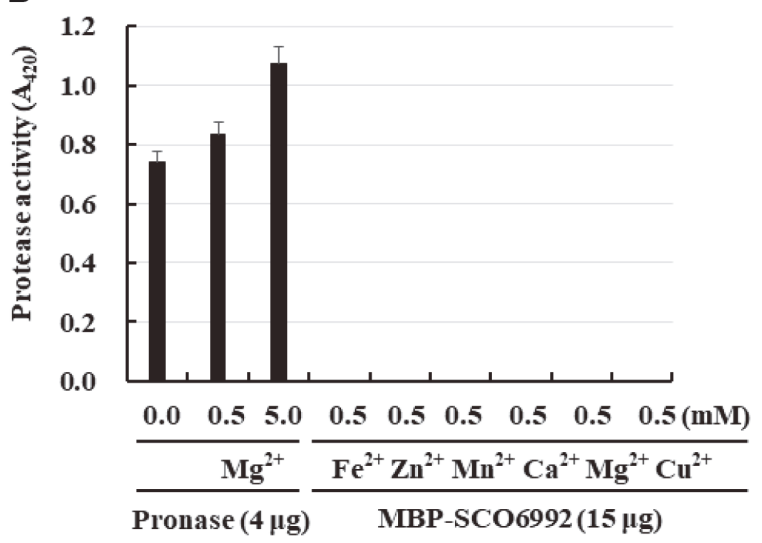

Fig. 5. Measurement of the protease activity of MBP-SCO6992. (A) Skim milk hydrolysis assay. To determine its total protease activity, purified MBP-SCO6992 aliquots were dropped onto a 1\% skim milk agar plate, and the formation of clear zones was observed after incubation at $37^{\circ} \mathrm{C}$ for $24 \mathrm{~h}$. (B) Azocasein hydrolysis assay. The total protease activity of MBPSCO6992 toward azocasein $(1 \%)$ was measured spectrophotometrically at $420 \mathrm{~nm}\left(\mathrm{~A}_{420}\right)$ after running a reaction at $37^{\circ} \mathrm{C}$ for $30 \mathrm{~min}$. The effect of metal ions on protease activity was also investigated by adding various metal ions to the reaction mixture. In both (A) and (B), pronase, a commercially available protease mixture, was used as the positive control. All experiments were repeated at least 3 times, and their average values were calculated.

However, after reacting with $\mathrm{Zn}^{2+}$, the intensity of the $\mathrm{CD}$ band within this wavelength range decreased, suggesting that the secondary structure of the protein had changed after binding $\mathrm{Zn}^{2+}$ (Fig. 4B). Furthermore, no significant changes in the $\mathrm{CD}$ spectrum were observed that could indicate that the protein was binding any other of the metal ions tested $\left(\mathrm{Fe}^{2+}, \mathrm{Mn}^{2+}, \mathrm{Mg}^{2+}\right.$ ) (data not shown). These data suggested that SCO6992 might be a zincbinding metalloprotein.

\section{Analysis of the Protease Activity of the MBP-SCO6992 Protein}

Based on in silico analyses and the confirmation of its zinc-binding ability, SCO6992 was presumed to be a zincdependent metalloprotease. In a protease activity test (Fig. 5A), pronase, a commercially available protease mixture, showed a large hydrolysis zone in skim milk agar plate tests, whereas SCO6992 did not show any hydrolysis activity. When the protease activity was measured toward azocasein, pronase also showed a strong activity that increased by the presence of $\mathrm{Mg}^{2+}$. In contrast, no protease activity was shown by SCO6992 regardless of the presence or absence of metal ions, including $\mathrm{Zn}^{2+}$ (Fig. 5B). The above reactivity trend was the same for other substrates such as artificial substrates for trypsin and chymotrypsin (data not shown). Therefore, we determined that SCO6992 was not a metalloprotease.

\section{$\beta$-Glucuronidase Activity of SCO6992}

In an attempt to identify any enzymatic activity of the SCO6992 protein, we used an API ZYM kit, which analyzes 19 different enzymatic activities, and is routinely used for microbial identification. As a result, a weak acid phosphatase activity and a strong $\beta$-glucuronidase activity were detected in MBP-SCO6992 (Fig. 6A). To confirm these results, we further searched for acid phosphatase activity using 2-naphthyl phosphate, an artificial substrate, but no activity was observed regardless of the presence or absence of $\mathrm{Zn}^{2+}$ ions (data not shown). However, when measuring $\beta$-glucuronidase activity using $p$-nitrophenyl- $\beta$ - $D$-glucuronide as substrate, a strong activity was detected (Fig. 6B). Noteworthy, the $\beta$-glucuronidase activity of MBP-SCO6992 was slightly increased by the presence of $\mathrm{Zn}^{2+}$ ions, but the difference was not significant.

Moreover, a zymographic analysis on native polyacrylamide gel confirmed that the purified MBP-SCO6992 protein had $\beta$-glucuronidase activity on an artificial substrate such as AS-BI- $\beta$-D-glucuronide. Especially, the active band was not observed in the total protein samples obtained from E. coli JM109/pMAL-c2x, but was clearly observed in the total protein samples obtained from E. coli JM109/pMAL-c2x-SCO6992, implying that the $\beta$ glucuronidase activity of the transformant was caused by heterologous expression of MBP-SCO6992 (Fig. 6C). All these results suggest that SCO6992 is a protein having $\beta$-glucuronidase activity and has a great influence on the biosynthesis pathways of the secondary metabolism in S. coelicolor.

\section{Discussion}

S. coelicolor is the best characterized strain at both the molecular and genetic levels among the species of the genus Streptomyces. Because it can produce two kinds of pigmented antibiotics, mutants which simultaneously block the biosynthesis of more than one antibiotic can be easily selected with the naked eye, and thus several classes of mutants have been reported in S. coelicolor. For instance, a simultaneous loss of the capacity of synthesizing antibiotics and of morphological differentiation was reported in the bld mutant [3]. However, mutants like $a b s, a b a$, and $a f s$ are pleiotropically defective in antibiotic synthesis but have an almost normal 
A

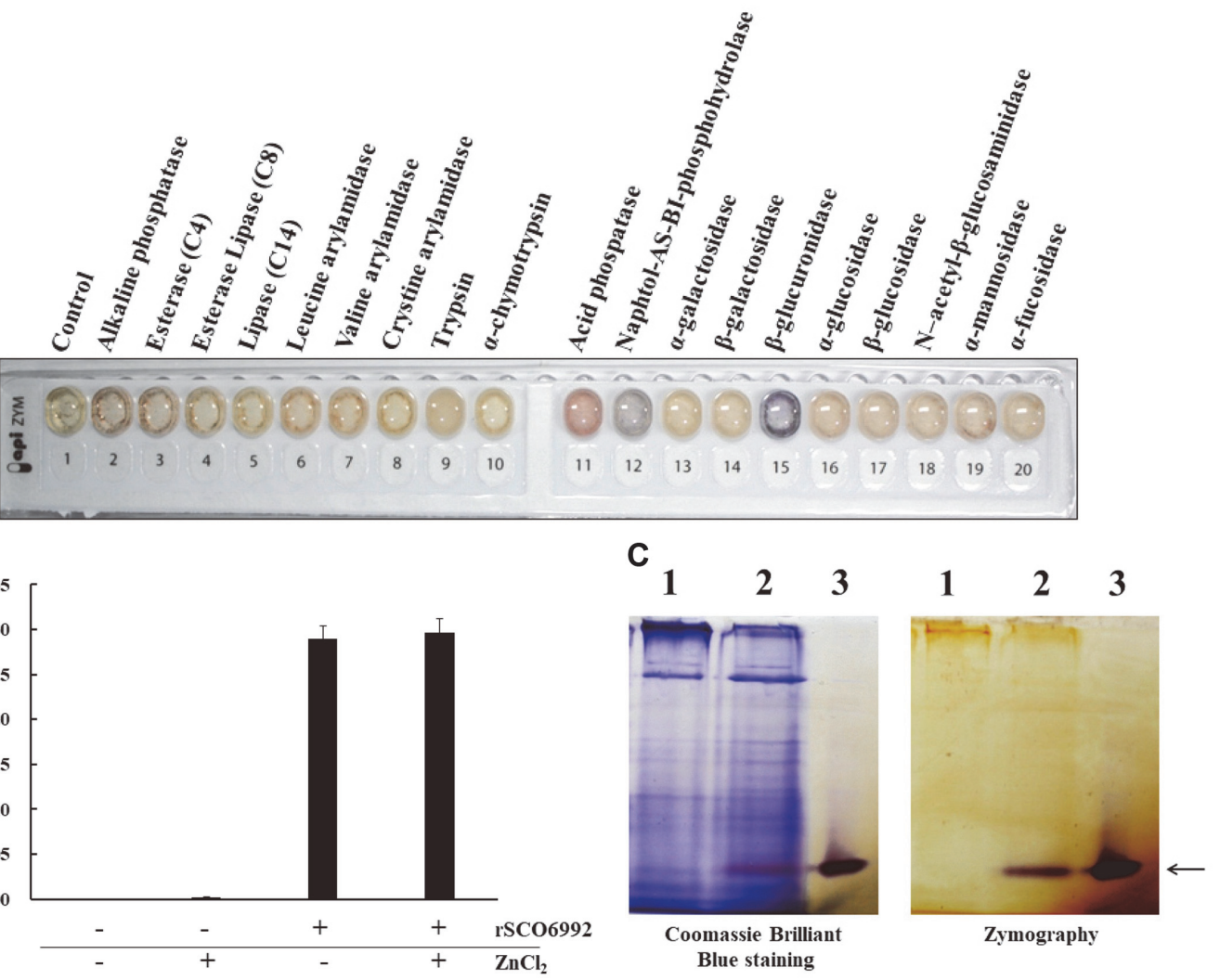

Fig. 6. $\beta$-glucuronidase activity of MBP-SCO6992. (A) Detection of $\beta$-glucuronidase activity. An API ZYM kit (bioMérieux) was used to investigate the enzymatic activity of the purified protein. MBP-SCO6992 showed a strong $\beta$ glucuronidase activity and a week acid phosphatase activity. (B) Measurement of $\beta$-glucuronidase activity. The artificial substrate $p$-nitrophenyl- $\beta$-D-glucuronide was used to measure $\beta$-glucuronidase activity. After incubating the enzymatic reaction at $37^{\circ} \mathrm{C}$ for $1 \mathrm{~h}$, the concentration of $p$-nitrophenol, a product of the hydrolysis, was measured at $415 \mathrm{~nm}\left(\mathrm{~A}_{415}\right)$. MBPSCO6992 showed strong hydrolyzing activity against the substrate used, and showed a slight increase in activity by the addition of $\mathrm{Zn}^{2+}$ ions. All experiments were repeated at least 3 times, and their average values were calculated. (C) Zymographic analysis for $\beta$-glucuronidase. The protein samples were electrophoresed onto a $9 \%$ native polyacrylamide gel. Then, the gel was immersed in $0.2 \mathrm{M}$ sodium acetate buffer ( $\mathrm{pH} 5.2,100 \mathrm{ml}$ ) containing $40 \mathrm{mg}$ of naphthol AS-BI- $\beta$-D-glucuronide (an artificial substrate) and $40 \mathrm{mg}$ of Fast Garnet. The color change on the zymogram was recorded. Lanes: 1, cell free extract of $E$. coli JM109/pMAL-c2x after IPTG induction; 2, E. coli JM109/pMAL-c2x-SCO6992 after IPTG induction; 3, purified SCO6992MBP. Both overexpressed and purified MBP-SCO6992 proteins show $\beta$-glucuronidase activity (indicated by an arrow).

morphological development $[7,4,23,24]$.

In pleiotropically defective mutants, mutations in a diversity of genes have been identified, for example, mutations in genes encoding sigma factors [25], DNA-binding proteins [26], tRNAs [27], RNase III [28], protein kinases [10], methyltransferases [29], adenylate cyclase [30], or S-Adenosyl-L-methionine synthetase [31], implicating that both the morphological and physiological differentiation of Streptomycetes are affected by various factors. Moreover, some global regulatory genes can function beyond species barriers. For example, the AbsA1/ AbsA2 and AfsQ1/AfsQ2 two-component protein systems are both composed of His-Asp kinases and exert their ability to stimulate antibiotic production in S. coelicolor as well as in S. lividans [14, 6, 32]. The global regulatory proteins, AfsR/AfsK, which are Ser/Thr kinases [12,33], and PtpA phosphotyrosine protein phosphatase [34] also exert similar effects on S. coelicolor and S. lividans.

In this study, we cloned a DNA fragment that restored the ability to produce four antibiotics in S. coelicolor abs8752, a new abs-type mutant [11]. Of the two putative genes in the clone, SCO6992 but not SCO6993 could stimulate antibiotic production in S. coelicolor strains. Furthermore, we observed that SCO6992 also stimulated the production of pigmented antibiotics in S. lividans TK24 to the same extent as in S. coelicolor (data not shown). Phylogenetically, S. lividans is known to be closely related to $S$. coelicolor, but the biosynthetic genes for actinorhodin and undecylprodigiosin commonly found in S. lividans are usually silent [23]. Like many regulatory genes, such as afsR, afsK, afs Q, absA, and $p t p A, S C O 6992$ highly induced antibiotic biosynthesis in S. lividans, leading us to conclude that SCO6992 played a global regulatory role in antibiotic production in both Streptomyces strains.

The presence of a metalloprotease domain with a putative metal binding motif led us to assume that SCO6992 
might be a metalloprotease. Given that several studies have reported that the expression of some proteases have a great influence on both morphological differentiation and the secondary metabolism of Streptomyces strains [17, 35], our hypothesis seemed plausible. However, no metalloprotease activity was detected in the purified MBPSCO6992 fusion protein. Instead, we concluded that SCO6992 is a $\beta$-glucuronidase through enzymatic and zymographic assays.

$\beta$-glucuronidases belong to the glycosidase family 2 of proteins, and are known to hydrolyze complex carbohydrates. In humans, $\beta$-glucuronidases hydrolyze glycosaminoglycans, releasing $\beta$-D-glucuronic acid residues from their non-reducing end. Increasing pathological evidence has indicated that $\beta$-glucuronidases in humans may be sensitive indicators for early identification of cell damage. In fact, an increase in $\beta$-glucuronidase levels has been reported in many pathological conditions, such as liver cirrhosis, inflammations, cholestatic jaundice, neoplasms, and tuberculosis [36].

Currently, functional studies on $\beta$-glucuronidases in prokaryotes seem to be very rare. The gusA gene (NP_416134) encoding $\beta$-glucuronidase (GUS) in E. coli has been widely used as a reporter gene to measure gene expression levels from specific promoters. However, studies on $\beta$-glucuronidase enzymes or D-glucuronic acid in Streptomyces are not available in Medline. Rather, it has been reported that Streptomyces does not have any GUS [37], implying that GusA homologues have not been found in this genus.

The amino acid sequences of SCO6992 (359 aa long) and GUS from E. coli (603 aa long) are not similar. Moreover, SCO6992 does not show any homology with any of the previously reported $\beta$-glucuronidases. Therefore, SCO6992 should be considered as a novel protein with $\beta$-glucuronidase activity. Interestingly, gus $A$ from $E$. coli has been frequently used as a reporter gene in S. lividans TK24 [37, 38] and S. coelicolor [39], however its ability to stimulate pigment production has never been reported.

We placed a paper disc soaked in D-glucuronic acid on a plate spread with spores of S. coelicolor and S. lividans, and searched for changes in pigment production while incubating it. However, no significant difference in color or growth was detected around the disc (Fig. S1). This suggests that the effect of SCO6992 on stimulating antibiotic production is not due to $\mathrm{D}$-glucuronic acid, but to other still unknown substrate modified by the enzyme.

Current knowledge cannot explain how the $\beta$-glucuronidase activity of the SCO6992 protein stimulates secondary metabolic pathways. Therefore, studies searching for the intracellular substrate of the SCO6992 protein and on the metabolic pathway linking such substrate with the biosynthesis of antibiotics are needed. These efforts will provide new clues to the functional identification of SCO6992 orthologues, presently listed as hypothetical proteins.

\section{Acknowledgments}

The Authors are extremely grateful to Dr. Charnpness in Michigan State Univ. for the Streptomyces coelicolor abs8752 strain. This research was supported by Basic Science Research Program through the National Research Foundation of Korea (NRF) funded by the Ministry of Science, ICT \& Future Planning (NRF-2020R1F1A1060789).

\section{Conflict of Interest}

The authors have no financial conflicts of interest to declare.

\section{References}

1. Lu T, Cao Q, Pang X, Xia Y, Xun L, Liu H. 2020. Sulfane sulfur-activated actinorhodin production and sporulation is maintained by a natural gene circuit in Streptomyces coelicolor. Microb. Biotechnol. 13: 1917-1932.

2. Liu G, Chater KF, Chandra G, Niu G, Tan H. 2013. Molecular regulation of antibiotic biosynthesis in Streptomyces. Microbiol. Mol. Biol. Rev. 77: 112-143

3. Chater KF. 2013. Curing baldness activates antibiotic production. Chem. Biol. 20: 1199-1200.

4. Brian P, Riggle PJ, Santos RA, Champness WC. 1996. Global negative regulation of Streptomyces coelicolor antibiotic synthesis mediated by an absA-encoded putative signal transduction system. J. Bacteriol. 178: 3221-3231.

5. Bentley SD, Chater KF, Cerdeño-Tárraga A-M, Challis GL, Thomson NR, James KD, et al. 2002. Complete genome sequence of the model actinomycete Streptomyces coelicolor A3(2). Nature 417: 141-147.

6. Ryding NJ, Anderson TB, Champness WC. 2002. Regulation of the Streptomyces coelicolor calcium-dependent antibiotic by absA, encoding a cluster-linked two-component system. J. Bacteriol. 184: 794-805.

7. Price B, Adamidis T, Kong R, Champness W. 1999. A Streptomyces coelicolor antibiotic regulatory gene, absB, encodes an RNase III homolog. J. Bacteriol. 181: 6142-6151.

8. Gravenbeek ML, Jones GH. 2008. The endonuclease activity of RNase III is required for the regulation of antibiotic production by Streptomyces coelicolor. Microbiology 154(Pt 11): 3547-3555.

9. Hesketh A, Kock H, Mootien S, Bibb M. 2009. The role of $a b s$ C, a novel regulatory gene for secondary metabolism, in zinc-dependent antibiotic production in Streptomyces coelicolor A3(2). Mol. Microbiol. 74: 1427-1444.

10. van der Heul HU, Bilyk BL, McDowall KJ, Seipke RF, van Wezel GP. 2018. Regulation of antibiotic production in Actinobacteria: new perspectives from the post-genomic era. Nat. Prod. Rep. 35: 575-604.

11. Park U, Suh JW, Hong SK. 2000. Genetic analysis of absR, a new abs locus of Streptomyces coelicolor. J. Microbiol. Biotechnol. 10: 169175.

12. Hong SK, Kito M, Beppu T, Horinouchi S. 1991. Phosphorylation of the AfsR product, a global regulatory protein for secondarymetabolite formation in Streptomyces coelicolor A3(2). J. Bacteriol. 173: 2311-2318.

13. Kieser H, Bibb MJ, Buttner MJ, Chater FK, Hopwood DA. 2000. Practical Streptomyces Genetics. The John Innes Foundation, Norwich, UK.

14. Anderson TB, Brian P, Champness WC. 2001. Genetic and transcriptional analysis of abs A, an antibiotic gene cluster-linked twocomponent system that regulates multiple antibiotics in Streptomyces coelicolor. Mol. Microbiol. 39: 553-566.

15. Vara J, Lewandowska-Skarbek M, Wang YG, Donadio S, Hutchinson CR. 1989. Cloning of genes governing the deoxysugar portion of the erythromycin biosynthesis pathway in Saccharopolyspora erythraea (Streptomyces erythreus). J. Bacteriol. 171: $5872-5881$. 
16. Tsevelkhorloo M, Kim SH, Kang DK, Lee CR, Hong SK. 2021. NADP ${ }^{+}$-dependent dehydrogenase SCO3486 and cycloisomerase SCO3480: key enzymes for 3,6-anhydro-L-galactose catabolism in Streptomyces coelicolor A3(2). J. Microbiol. Biotechnol. 31: 756763.

17. Choi SS, Chi WJ, Lee JH, Kang SS, Jeong BC, Hong SK. 2001. Overexpression of the sprD gene encoding Streptomyces griseus protease D stimulates actinorhodin production in Streptomyces lividans. J. Microbiol. 39: 305-313

18. Yuan D, Shen Z, Liu R, Chi Z, Zhu J. 2011. Study on the binding of cerium to bovine serum albumin. J. Biochem. Mol. Toxicol. 25: 263268.

19. Chern CJ. 1977. Detection of active heteropolymeric beta-glucuronidase in hybrids between mouse cells and human fibroblasts with beta-glucuronidase deficiency. Proc. Natl. Acad. Sci. USA 74: 2948-2952.

20. Medda S, Swank RT. 1985. Egasyn, a protein which determines the subcellular distribution of beta-glucuronidase, has esterase activity. J. Biol. Chem. 260: 15802-15808.

21. Marchler-Bauer A, Bryant SH. 2004. CD-Search: protein domain annotations on the fly. Nucleic Acids Res. 32: 327-331.

22. Baumann U, Wu S, Flaherty KM, McKay DB. 1993. Three-dimensional structure of the alkaline protease of Pseudomonas aeruginosa: a two-domain protein with a calcium binding parallel beta roll motif. EMBO J. 12: 3357-3364.

23. Horinouchi S. 2003. AfsR as an integrator of signals that are sensed by multiple serine/threonine kinases in Streptomyces coelicolor A3(2). J. Ind. Microbiol. Biotechnol. 30: 462-467.

24. Fernandez-Moreno MA, Martin-Triana AJ, Martinez E, Niemi J, Kieser HM, Hopwood DA, et al. 1992. abaA, a new pleiotropic regulatory locus for antibiotic production in Streptomyces coelicolor. J. Bacteriol. 174: 2958-2967.

25. López-García MT, Yagüe P, González-Quiñónez N, Rioseras B, Manteca A. 2018. The SCO4117 ECF sigma factor pleiotropically controls secondary metabolism and morphogenesis in Streptomyces coelicolor. Front. Microbiol. 9: 312.

26. Xu Z, Li Y. 2020. A MarR-family transcriptional factor MapR positively regulates actinorhodin production in Streptomyces coelicolor. FEMS Microbiol. Lett. 367: fnaal40.

27. Bhatia SK, Lee BR, Sathiyanarayanan G, Song HS, Kim J, Jeon JM, et al. 2016. Biomass-derived molecules modulate the behavior of Streptomyces coelicolor for antibiotic production. 3Biotech. 6: 223.

28. Xu W, Huang J, Cohen SN. 2008. Autoregulation of AbsB (RNase III) expression in Streptomyces coelicolor by endoribonucleolytic cleavage of absB operon transcripts. J. Bacteriol. 190: 5526-5530.

29. Pisciotta A, Manteca A, Alduina R. 2018. The SCO1731 methyltransferase modulates actinorhodin production and morphological differentiation of Streptomyces coelicolor A3(2). Sci. Rep. 8: 13686.

30. Nitta K, Carratore FD, Breitling R, Takano E, Putri SP, Fukusaki E. 2020. Multi-omics analysis of the effect of cAMP on actinorhodin production in Streptomyces coelicolor. Front. Bioeng. Biotechnol. 8: 595552.

31. Kim DJ, Huh JH, Yang YY, Kang CM, Lee IH, Hyun CG, et al. 2003. Accumulation of S-adenosyl-L-methionine enhances production of actinorhodin but inhibits sporulation in Streptomyces lividans TK23. J. Bacteriol. 185: 592-600.

32. Shu D, Chen L, Wang W, Yu Z, Ren C, Zhang W, et al. 2009. afsQ1-Q2-sigQ is a pleiotropic but conditionally required signal transduction system for both secondary metabolism and morphological development in Streptomyces coelicolor. Appl. Microbiol. Biotechnol. 81: 1149-1160.

33. Tomono A, Mashiko M, Shimazu T, Inoue H, Nagasawa H, Yoshida M, et al. 2006. Self-activation of serine/threonine kinase AfsK on autophosphorylation at threonine-168. J. Antibiot. 59: 117-123.

34. Umeyama T, Tanabe Y, Aigle BD, Horinouchi S. 1996. Expression of the Streptomyces coelicolor A3(2) ptpA gene encoding a phosphotyrosine protein phosphatase leads to overproduction of secondary metabolites in S. lividans. FEMS Microbiol. Lett. 144: $177-184$

35. Demir Z, Bayraktar A, Tunca S. 2019. One extra copy of lon gene causes a dramatic increase in actinorhodin production by Streptomyces coelicolor A3(2). Curr. Microbiol. 76: 1045-1054.

36. Wang P, Jia Y, Wu R, Chen Z, Yan R. 2021. Human gut bacterial $\beta$-glucuronidase inhibition: An emerging approach to manage medication therapy. Biochem. Pharmacol. 190: 114566.

37. Paradis FW, Shareck F, Dupont C, Kluepfel D, Morosoli R. 1996. Expression and secretion of beta-glucuronidase and Pertussis toxin S1 by Streptomyces lividans. Appl. Microbiol. Biotechnol. 45: 646-651.

38. Boukhris I, Dulermo T, Chouayekh H, Virolle MJ. 2016. Evidence for the negative regulation of phytase gene expression in Streptomyces lividans and Streptomyces coelicolor. J. Basic Microbiol. 56: 59-66.

39. Rudolph MM, Vockenhuber MP, Suess B. 2015. Conditional control of gene expression by synthetic riboswitches in Streptomyces coelicolor. Methods Enzymol. 550: 283-299. 